\title{
De L'Innovation Technologique et Sociale dans les Mecanismes de Declaration des Naissances et Deces dans les Delais chez les populations de la Region de la Nawa (Cote d'Ivore)
}

\author{
Affessi Affessi, \\ Diakalidia Konate, \\ Etat civil, ONI, Côte d'Ivoire \\ Adon Simon Affessi, \\ Bosson Jean Fernand Mian, \\ Université Peleforo Gon Coulibaly de Korhogo, Côte d'Ivoire \\ Koffi Pascal Sialou \\ Etat civil, ONI, Côte d'Ivoire
}

Doi:10.19044/esj.2019.v15n22p83～URL:http://dx.doi.org/10.19044/esj.2019.v15n22p83

\section{Résumé}

Cette étude s'est intéressée aux nouveaux mécanismes de déclaration des naissances et décès dans les délais en Côte d'Ivoire. A travers le cas pratique de la région des populations de la Nawa, au Sud-Ouest du pays, la présente étude socio-anthropologique vise à tester la portée sociale de cette innovation technologique dans le système d'enregistrement des faits d'état civil en général, et particulièrement, la dimension des naissances et décès. Elle s'est déroulée de Février à Mai 2018 et a mobilisé un échantillon de deuxcent-cinq (205) participants, constitués d'autorités administratives, coutumières et populations des localités cibles. Cette population d'enquête a été choisie selon les techniques d'échantillonnage accidentel et de choix raisonné et investiguée à l'aide de questionnaires et de guides d'entretien. Les résultats ont révélé que la plupart des populations ont marqué leur adhésion aux nouveaux mécanismes de déclaration des naissances et décès dans les délais en ce sens que, désormais, c'est l'institutionnel qui converge vers le social (ou l'administration de l'état civil qui converge vers les populations) par l'entremise des Points de Collecte Communautaires (PCC) et Points de Collecte Sanitaires (PCS), chose qui autrefois, n'existait pas. Ainsi, l'on note à titre de retombée positive, la maitrise des naissances et décès survenant en temps réel dans la zone d'étude mais également et surtout, ces nouveaux mécanismes ont permis de contourner les formes de tracasseries dans la déclaration des faits d'état civil. 
Mots-clés : Innovation, Mécanisme, Déclaration, Naissances, Décès, Soubré

\title{
Technological and Social Innovation in Mechanisms of Declaration of Births and Deaths within the Periods of Populations in Nawa Region (Cote d'Ivore)
}

\author{
Affessi Affessi, \\ Diakalidia Konate, \\ Etat civil, ONI, Côte d'Ivoire \\ Adon Simon Affessi, \\ Bosson Jean Fernand Mian, \\ Université Peleforo Gon Coulibaly de Korhogo, Côte d'Ivoire \\ Koffi Pascal Sialou \\ Etat civil, ONI, Côte d'Ivoire
}

\begin{abstract}
This paper focuses on new mechanisms for the timely registration of births and deaths in Côte d'Ivoire. Through the practical case of the Nawa people's region in the southwest of the country, this socio-anthropological study aims to test the social significance of this technological innovation in the civil registration system in general, and in particular, the birth and death dimension. It took place from February to May 2018 and involved a sample of two hundred and five (205) participants, which was made up of administrative authorities, customary authorities, and populations from the target localities. This survey population was selected using accidental sampling and reasoned choice techniques. The study was carried out using questionnaires and interview guides. The results revealed that most populations have expressed their support for the new mechanisms for the timely declaration of births and deaths. This means that, presently, it is the institution that converges towards social issues (or civil status administration that converges towards populations) through Community Collection Points (CCPs) and Health Collection Points (HCPs). This, however, has not existed before. Thus, as a positive outcome, the control of births and deaths occurring in real time in the study area is noted. Above all, these new mechanisms have made it possible to bypass the forms of harassment in the declaration of civil status facts.
\end{abstract}


Keywords: Innovation, Mechanism, Declaration, Births, Deaths, Soubré

\section{Introduction}

En Côte d'Ivoire, la déclaration des faits d'état civil constitue encore aujourd'hui un problème pour la société ivoirienne, à telle enseigne qu'elle intègre les préoccupations majeures en matière de politique de l'Etat. En effet, la production des statistiques des faits d'état civil qui reste encore peu fiable, incomplète et insuffisamment exploitée en raison du faible taux observé dans la déclaration des faits d'état civil (pouvant amener à la consolidation d'une base de données nationale relative aux faits d'état civil) constitue des entraves à l'élan du pays vers une modernisation du système d'état civil. Même si cet état de fait est imputable dans une certaine mesure aux crises sociopolitiques entre 2002 et 2011 qui ont fortement perturbé le pays et affecté durablement tous les secteurs de l'État, en particulier celui du système d'enregistrement des faits d'état civil (le non enregistrement ou le retard dans l'enregistrement des faits d'état civil, la destruction des centres d'état civil et des registres pendant les périodes critiques dans certaines régions), il n'en demeure pas moins qu'en dépit des nombreuses campagnes de sensibilisations et d'opérations d'audiences foraines qui sont menées, on constate que de nombreuses populations et certains acteurs impliqués dans le processus d'enregistrement des faits d'état civil ignorent encore l'utilité de la déclaration des naissances et décès dans les délais (Rapport d'étude diagnostique de l'Etat Civil en Côte d'Ivoire, Juin 2015). Pour faire face à cette situation, des mesures importantes ont été prises par le Gouvernement ivoirien après la crise militaro-politique de 2002, pour moderniser le système d'état civil. Ainsi, a été mis en place le programme de Modernisation de l'Etat Civil en Côte d'Ivoire (MEECI). Ces diverses mesures ont été appuyées par des Partenaires Techniques et Financiers (PTF) tels que l'Union Européenne, l'UNICEF, l'UNHCR, l'ONUCI, l'USAID, l'AIMF etc. Ces efforts conjoints ont permis d'améliorer quelque peu le taux d'enregistrement des naissances. (Rapport d'étude diagnostique de l'Etat Civil en Côte d'Ivoire, Juin 2015 op.cit.). Ainsi, le taux d'enregistrement a évolué passant de 2015 à 2016 respectivement à 72,8\% et $74,8 \%$. Malgré ces efforts consentis, l'évaluation complémentaire du système d'état civil ivoirien s'est faite suivant l'approche de Africa Programme on Accelerated Improvement of Civil Registration and Vital Statistics /Programme Africain pour l'Amélioration des systèmes d'enregistrement et des statistiques d'état civil (APAI-CRVS), qui a été présentée par la Conférence des Ministres Africain chargés de l'enregistrement des faits d'état civil ( 2017), () Cette approche fait remarquer que le système d'état civil ivoirien est classé «faible » dans la typologie des systèmes nationaux d'enregistrement des faits d'état civil en Afrique (Rapport SNECI, 2017). Cet 
état des faits va conduire des experts nationaux appuyés d'experts internationaux et de partenaires techniques et financiers à des travaux d'élaboration d'une Stratégie Nationale de l'Etat Civil et de l'Identification. Ce document stratégique est le résultat de la volonté politique du Gouvernement ivoirien de se doter d'un cadre de référence pour asseoir un système d'état civil fiable, sécurisé et statistiquement utile pour l'Etat.

Par ailleurs, étant donné que le système d'enregistrement des faits d'état civil sur support papier (registre) est extrêmement vulnérable et leur exploitation et reconstitution fastidieuses et onéreuses, il est apparu important d'évoluer vers un système d'état civil informatisé débouchant sur un identifiant unique qui faciliterait la mise en place d'un Registre National des Personnes Physiques. Il s'agit là, d'une nouvelle politique de modernisation des faits d'état civil en Côte d'Ivoire qui s'opérationnalise et s'expérimente à travers cette étude sur les nouveaux mécanismes de déclaration des naissances et décès dans les délais. Celle-ci part de l'idée que la non déclaration de toutes les naissances et des décès entrainent des difficultés quant à la maitrise des variables sociodémographiques indispensables en matière de planification de développement.

Ainsi, à l'aide du cas pratique de la région de la Nawa, la présente recherche intitulée: "De l'innovation technologique et sociale dans les mécanismes de déclaration des naissances et décès dans les délais dans la région de la Nawa au Sud-Ouest de la Côte d'Ivoire », intègre le projet pilote de la promotion des nouveaux mécanismes de déclaration des naissances et décès dans les délais. Notre recherche compte relever certains défis majeurs notamment, rehausser le taux de déclaration des naissances et décès dans les délais, rapprocher l'administration de l'état civil des populations par la création des points de collecte dans tous les villages et centres de santé, et la mise en place de bureaux d'état civil.

Il s'agit en définitive de rendre le système de l'état civil ivoirien plus compétitif et adapté aux normes internationales et d'en faire un modèle dans la sous-région dans l'atteinte du taux de déclaration universel.

Dès lors, le questionnement ci-dessous mérite d'être analysé. Comment sommes-nous arrivés aux nouveaux mécanismes de déclaration des naissances et décès dans les délais ? Quelles sont ces nouveaux mécanismes ainsi que leur particularité ou spécificité dans la déclaration des naissances et décès dans les délais ? Quelle est la portée sociale des mécanismes innovants dans la déclaration des naissances et décès dans les délais de la région de la Nawa ?

\section{I-Methodologie}

\section{I-1-Site et population de l'étude}

La présente étude mixte (qualitative et quantitative) s'est déroulée au Sud-Ouest du pays dans les quatre (04) départements de la région de la Nawa 
(Soubré) à savoir Soubré, Méagui, Guéyo et Buyo. Le choix de cette région est dû au fait que celle-ci fait partie des régions à faible taux de déclaration des naissances et de décès comparé aux autres régions du pays. Ce taux se situe à moins de $40 \%$ par rapport à une moyenne nationale qui est de $70 \%$. La collecte des données s'est effectuée plus précisément dans les SousPréfectures de Soubré, Grand-Zattry, Liliyo, Okrouyo, Buyo, Guéyo, Dabouyo, Méagui, Oupoyo dans la période de Février à Mai 2018 et a mobilisé deux-cent-cinq (205) participants. Ces (205) personnes enquêtées ont été sélectionnées selon la technique de choix raisonné c'est-à-dire le choix des éléments est basé sur le jugement du chercheur par rapport à leur caractère typique ou atypique et à l'aide de la technique d'échantionnage accidentel là où les éléments sont choisis au fur et à mesure qu'ils se présentent, guidée par le hasard des circonstances (Dufour \& Larivière, 2012). En effet, vue que les caractéristiques initiales de la population de départ de la zone d'enquête n'étant pas connues, alors les premières personnes rencontrées fortuitement (individus présents) lors de la visite de terrain de l'équipe de recherche dans 187 villages des 9 sous-préfectures ayant abrité l'étude ont été toutes choisies et intérrogées.

Vue cartographique de la zone d'étude avec les localités cibles

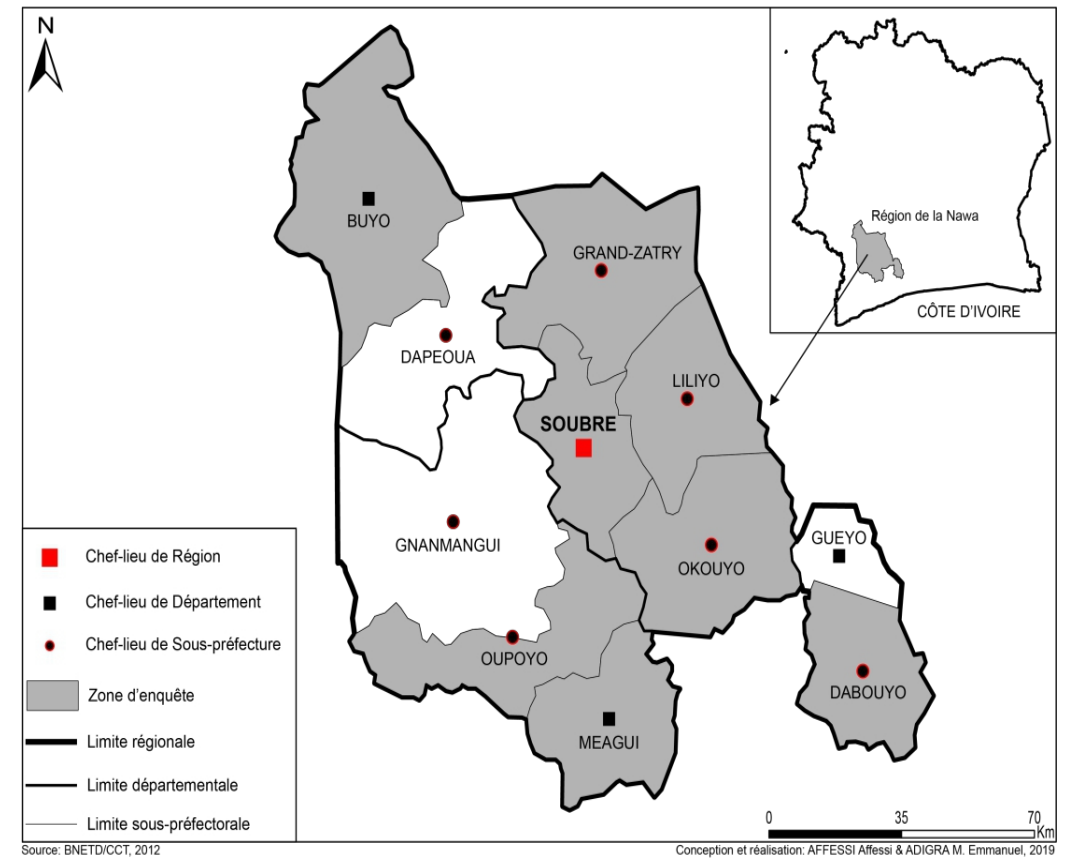

La population cible d'enquête était généralement constituée d'un point de vue sociologique, d'autochtones, allochtones et allogènes des localités choisies. Il s'agit spécifiquement d'acteurs administratifs ou non et d'acteurs coutumiers 
concernés directement ou indirectement par l'étude. Plus concrètement ces acteurs étaient composés de :

- Officiers d'état civil (Sous-préfets et maires);

- Tous les chefs de village ;

- Personnel de santé (DR Santé, DD Santé, médecins, infirmiers, sagesfemmes, aides-soignants);

- Leaders religieux résidents (Imams, Pasteurs, Prêtres);

- Leaders et Agents communautaires résidents (les chefs des communautés ethniques, les présidents de jeunes et de femmes, les chefs d'association ou de groupements coopératifs, ...).

\section{I-2-Collecte des données}

Avant la collecte des données proprement dite, une méthode d'approche destinée à toucher la population cible a été faite. Elle avait pour but de mobiliser la population et créer en elle un intérêt non seulement pour l'étude mais singulièrement obtenir son adhésion vis à vis des nouveaux mécanismes de déclaration. La méthode a consisté à mobiliser la population à travers plusieurs phases. Une campagne d'information et de communication média et hors média a été suivie de sessions de formation des acteurs directement impliqués. Ainsi, des affiches d'informations conçues ont été placées dans toutes les localités de l'étude, les radios de proximité ont été sollicitées pour diffuser des spots et communiquer sur le projet. En outre, un film institutionnel conçu et les articulations du projet ainsi que le rôle des différents acteurs ont été présentés.

Le recueil des données a été faite grâce à la mobilisation des techniques d'entretien et de questionnaire. Les données ont été collectées à partir des formulaires à deux feuillets de déclaration de naissance ou de décès, de registres d'ordre pour les naissances et les décès. Ces outils mobilisés peuvent se résumer ainsi :

- Les formulaires de déclaration des naissances et décès à deux feuillets pour PCC et PCS ;

- Les registres de naissance et de décès ;

- Les registres mains ;

- Les bordereaux de transmission ;

- Le Kit de matériel (boîtes d'archive, chemises à rabat, marqueurs, fluors...).

Les données recueillies sur les formulaires ont servi à renseigner les registres d'état civil (registres de décès et de naissances). Elles ont été saisies à l'ordinateur dans un logiciel dédié à l'état civil, à partir duquel sont faites les impressions des actes de naissances et décès (extraits et copies intégrales d'acte de naissance et de décès). 
Par ailleurs, les procédés d'analyse de contenu et d'analyse statistiques (présentation de tableau, de diagramme et d'histogramme) générées sur Excel ont constitué d'une façon générale la méthode d'analyse des données collectées dans le cadre de cette étude. L'étude s'inscrit dans le champ de la sociologie de l'innovation et du développement communautaire. A cet effet, l'approche théorique de l'innovation de Marty (1955) semble pertinente dans l'explication de l'introduction des mécanismes innovants dans la déclaration des naissances et décès dans les délais en Côte d'Ivoire à l'aide du cas de la région de la Nawa. Selon cette approche, l'innovation se caractérise par la mise en vigueur de nouvelles fonctions productrices, les PCC et PCS dans le cas de notre étude, jouent ce rôle dans l'enregistrement des faits d'état civil en Côte d'Ivoire.

\section{II-Resultats}

\section{II-1- Contexte d'emergence des nouveaux mecanismes de declaration des naissances et deces dans les delais}

L'avènement des nouveaux mécanismes de déclaration des faits d'état civil en général et plus particulièrement des naissances et décès résulte des insuffisances majeures décriées dans les formes anciennes de déclaration ou d'enregistrement des faits d'état civil. Ces faits d'état civil étaient obstrués par diverses barrières qui s'identifiaient au niveau des populations, mais également au niveau des différentes institutions qui sont impliquées dans le processus. Ces barrières qui constituent des facteurs freinant l'enregistrement systématique des naissances et décès à l'état-civil se perçoivent à deux grands niveaux.

Le premier groupe de facteurs est lié à la demande de services d'étatcivil. En effet, le faible recours des populations aux services d'état-civil pour la déclaration des naissances dans les délais est un des facteurs clés qui entrave l'enregistrement universel. Ce comportement des populations s'explique par plusieurs raisons dont l'ignorance à la fois de l'intérêt pour eux et pour l'Etat à déclarer les faits d'état civil mais également des dispositions légales relatives à la déclaration des naissances et décès et la persistance de certains pesanteurs socioculturelles. Ces facteurs agissent négativement sur la demande de services d'état-civil.

Le second groupe de facteurs est lié à l'offre de service d'état-civil c'est-à-dire à certains dysfonctionnements et pratiques en cours au niveau des centres d'état civil. Certaines pratiques non conformes aux dispositions légales des services dissuadent dans certains cas, la déclaration des naissances à l'état-civil (exigence de présentation de pièces d'identité des parents au moment de la déclaration, paiement de frais autres que les frais de timbres au moment de la déclaration, la faible qualification du personnel des services d'état-civil, les ruptures en intrants nécessaires à l'enregistrement des 
naissances, la faible supervision des services par la tutelle et l'autorité judiciaire).

Les conséquences de ces facteurs, d'un point de vue statistique, sont révélatrices du risque social que court l'état civil ivoirien. En effet, selon les résultats de l'Enquête Démographique et de Santé 2012 (EDS, 2012), 24\% des enfants de moins de 18 ans vivant en Côte d'Ivoire ne sont pas déclarés à l'étatcivil. Ce taux est de $35 \%$ chez les enfants de moins de 5 ans. Les données révèlent également que l'enregistrement des naissances des enfants est plus faible dans certaines régions du pays telles que le sud-ouest (54\%), l'ouest $(63 \%)$, le nord-ouest $(69 \%)$, le centre et le nord $(67 \%)$.

Les données de routine du Ministère de l'Intérieur sur l'enregistrement des nouvelles naissances au cours des trois années (2012-2014) montrent que près de la moitié des enfants nés entre 2012 et mai 2014 n'ont pas été déclarés à l'état civil dans les délais légaux (taux de déclaration des nouvelles naissances de $50 \%$ en $2012,47 \%$ en $2013,51 \%$ sur la période janvier-mai 2014). Ainsi, plus de la moitié des naissances enregistrées de 2012 à mai 2014 (54\%) ont été déclarées grâce à des mécanismes dérogatoires (loi spéciale, jugements supplétifs).

Dans un tel contexte, le faible taux de déclaration des nouvelles naissances et décès entraînerait indéniablement une baisse du taux d'enregistrement des naissances dans les années à venir. Cette situation pose inexorablement des problèmes d'accès à l'identité pour les enfants non déclarés, accroissant ainsi les risques d'apatridie.

La recherche de méthodes innovantes de déclarations reste indispensable et salutaire.

\section{II-2- Presentation et caracteristiques des nouveaux mecanismes de declaration des naissances et deces dans les delais}

II-2-1- Présentation des nouveaux mécanismes de déclaration des naissances et décès dans les délais

Les nouveaux mécanismes de déclaration des naissances et décès s'inscrivent dans une dynamique de renforcement des capacités techniques et matérielles des services d'état civil en matière de déclaration et d'enregistrement. Il s'agit d'une mise en place des mécanismes communautaires de déclaration des naissances et décès, d'une approche participative ou les populations sont au centre du processus. Le fait innovant provient du fait que c'est plutôt l'administration qui se déplace vers ses populations au travers de ses nouveaux organes mis en place. On assiste désormais non plus à un processus socio-institutionnel mais plutôt «"institutiono"social », en ce sens que l'institution de l'état civil converge désormais vers les populations urbaines et rurales même dans les espaces sociaux les plus reculés. 
Dans ces conditions, le phénomène d'accouchement à domicile par exemple, les démotivations ou découragement des populations pour cause de distance, de temps et de frais de déplacement ou autres faux frais ne s'érigent plus comme obstacle à l'enregistrement des nouvelles naissances et des décès à l'état civil.

Ces organes innovateurs de déclaration des naissances et décès dans les délais sont : les Points de Collecte Communautaires (PCC), les Points de Collecte Sanitaires (PCS), les animateurs de Bureau d'Etat Civil (BEC) pour la déclaration des naissances et des décès, les Bureaux Principaux (BP) de la sous-préfecture ou de la mairie, puis le Bureau de Centralisation des Données, représenté par l'ONI. Quelles sont donc leurs spécificités?

\section{II-2-2- Caractéristiques des nouveaux mécanismes de déclaration des naissances et décès dans les délais \\ Les Points de Collecte Communautaire (PCC)}

Les PCC sont représentés dans toutes les localités cibles de notre région d'intervention (la Nawa) particulièrement dans tous les villages. Il s'agit pour les agents coptés par l'ONI sur proposition du chef de village et validation de l'officier d'état civil (sous-préfet, maire), d'enregistrer sur des formulaires à deux volets puis dans un registre d'ordre, les naissances et les décès qui surviennent dans leur zone de compétence. Ces informations collectées sont apportées au BEC, équipés en outils informatiques et logiciel pour enregistrer les données. Ce dernier inscrit également les données dans des registres de naissance et de décès.

\section{Les Points de Collecte Sanitaires (PCS)}

Les PCS sont présents dans tous les centres de santé de la zone d'étude. Ils sont animés par le corps médical (sage femmes, infirmiers...). Les informations recueillies à ce niveau, sur les formulaires de déclaration de naissance ou de décès à deux volets, sont acheminées vers les BEC respectifs.

\section{Les Bureaux d'Etat Civil (BEC)}

Les BEC sont en fait les anciens centres secondaires, équipés pour constituer de véritables bureaux d'état civil. Leur rôle est de transcrire et d'enregistrer non seulement dans les registres de naissance ou de décès mais également à l'ordinateur dans un logiciel, les données issues des PCC et PCS et d'acheminer ces données sous la forme numérique et physiques vers le Bureau Principal d'état civil de la sous-préfecture ou la mairie. Notons que de nouveaux BEC sont créés pour être plus proches des PCC et PCS et faciliter leur déplacement. 


\section{Le Bureau Principal d'état civil}

Le bureau principal demeure le centre d'état civil de la sous-préfecture ou de la mairie qui réceptionne toutes les données issues des BEC. Ces données centralisées par circonscription d'état civil sont ensuite transmises à l'ONI qui est le Bureau de Centralisation des Données Nationales.

\section{Le Bureau de Centralisation des Données}

Le Bureau de Centralisation des Données ou bureau de coordination est l'Office National d'Identification qui réceptionne les données numériques provenant des Centre Principaux et assure le contrôle de l'opération sur toute l'étendue de la zone de l'opération pilote..

\section{II-3 Pertinence des Mecanismes innovants de declaration des naissances et deces dans les delais}

III-3-1 Points de Collecte Communautaires (PCC) et Points de Collecte Sanitaires (PCS), comme organes à promouvoir dans la déclaration des naissances et décès dans les délais

III-3-1-1- Efficacité et Pertinence sociale des PCC et PCS dans la captation des données de naissances dans les délais

Les points de collecte communautaires et sanitaires ont eu un véritable impact en terme de captation des naissances dans les délais sur l'ensemble des Sous-Préfectures où l'étude à été réalisée. Cela se perçoit à travers la réprésentation statistique ci-dessous.

Tableau 1. Synthèse des données des naissances par Sous-préfecture

NAISSANCE

\begin{tabular}{|c|c|c|c|c|c|c|c|c|c|c|c|c|}
\hline \multirow[b]{2}{*}{ LOCALITES } & \multicolumn{2}{|c|}{ FEV } & \multicolumn{2}{|c|}{ MARS } & \multicolumn{2}{|c|}{ AVRIL } & \multicolumn{2}{|c|}{ MAI } & \multicolumn{2}{|c|}{ SOUS TOTAL } & \multirow[t]{2}{*}{ TOTAL } & \multirow{2}{*}{$\begin{array}{c}\text { Moyenne } \\
\text { mensuelle sur } \\
\text { les } 4 \text { mois } \\
\end{array}$} \\
\hline & $\mathbf{H}$ & $\mathbf{F}$ & $\mathbf{H}$ & $\mathbf{F}$ & $\mathbf{H}$ & $\mathbf{F}$ & $\mathbf{H}$ & $\mathbf{F}$ & $\mathbf{H}$ & $\mathbf{F}$ & & \\
\hline SOUBRE & 112 & 93 & 113 & 104 & 119 & 92 & 93 & 64 & 437 & 353 & 790 & 197,5 \\
\hline GRAND ZATTRY & 114 & 112 & 106 & 72 & 79 & 71 & 103 & 85 & 402 & 340 & 742 & 185,5 \\
\hline LILIYO + MAYO & 115 & 106 & 143 & 125 & 89 & 98 & 102 & 111 & 449 & 440 & 889 & 222,25 \\
\hline OKROUYO & 86 & 84 & 87 & 94 & 63 & 64 & 40 & 47 & 276 & 289 & 825 & 206,25 \\
\hline MEAGUI & 170 & 152 & 183 & 135 & 117 & 127 & 147 & 126 & 617 & 540 & 1157 & 289,25 \\
\hline OUPOYO & 131 & 143 & 230 & 279 & 133 & 126 & 84 & 122 & 578 & 670 & 1248 & 312 \\
\hline GUEYO & 45 & 55 & 66 & 92 & 70 & 53 & 41 & 38 & 222 & 238 & 571 & 142,75 \\
\hline DABOUYO & 151 & 120 & 142 & 89 & 114 & 97 & 83 & 94 & 490 & 400 & 890 & 222,5 \\
\hline BUYO & 207 & 172 & 141 & 129 & 126 & 127 & 77 & 73 & 551 & 501 & 1052 & 263 \\
\hline $\begin{array}{c}\text { SOUS TOTAL } \\
\text { NAISSANCES } \\
\text { HOMME ET FEMME } \\
\end{array}$ & 1131 & 1037 & 1211 & 1119 & 910 & 855 & 770 & 760 & 4022 & 3771 & 8164 & 2041 \\
\hline $\begin{array}{c}\text { TOTAL } \\
\text { NAISSANCES } \\
\end{array}$ & \multicolumn{2}{|c|}{2168} & \multicolumn{2}{|c|}{2330} & \multicolumn{2}{|c|}{1765} & \multicolumn{2}{|c|}{1530} & \multicolumn{2}{|c|}{$7793+371$} & & \\
\hline Pourcentage & \multicolumn{2}{|c|}{$26,55 \%$} & \multicolumn{2}{|c|}{$28,53 \%$} & \multicolumn{2}{|c|}{$21,61 \%$} & \multicolumn{2}{|c|}{$18,74 \%$} & \multicolumn{2}{|c|}{$95,64 \%+4,54 \%$} & & \\
\hline
\end{tabular}

Source : Notre enquête de Février à Mai, 2018

NB : Parmi les 8164, il y a 371 naissances dont le sexe n'est pas spécifié à Okrouyo et Guéyo

Il ressort du tableau ci-dessus que sur l'ensemble des neuf (9) souspréfectures de la région de la Nawa, les PCC et PCS ont ensemble enregistré 
durant les quatre mois considérés, 8.164 déclarations dans les délais de Février à mai 2018 soit une moyenne de 2.041 déclarations par mois dans la région. Les PCC et PCS de la sous-préfecture de Oupoyo ont enregistré le plus fort effectif de naissances sur cette période suivie de la Sous-préfecture de Méagui et de Buyo avec respectivement 1.248 naissances, 1.157 et 1.052 soit une moyenne mensuelle de naissance de $312 ; 289$ et 263. Sur les 9 souspréfectures, les PCC et PCS de la sous-préfecture de Guéyo ont enregistré le plus faible effectif de naissances avec 571 naissances suivies de la Souspréfecture de Grand Zattry avec 742 naissances, soit (18\%) suivi par Méagui (18\%) et Oupoyo (17\%).

\section{III-3-1-2- Efficacité et Pertinence sociale des PCC et PCS dans la captation des données de décès dans les délais}

$\mathrm{Au}$ niveau de l'enregistrement des décès, les PCC et PCS ont également été très déterminants dans les Sous-Préfectures ayant abritées notre étude.

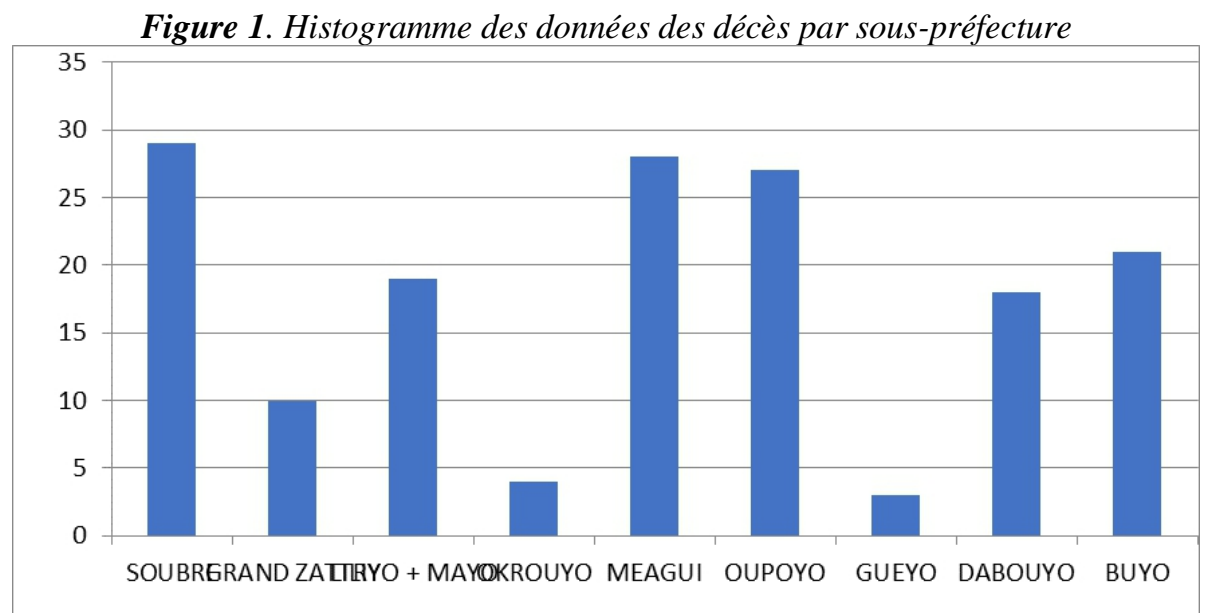

Source : Notre enquête de Février à Mai, 2018

L'on observe que les faibles déclarations de décès sont enregistrées à Guéyo (2\%) Okrouyo (3\%) et Grand-Zattry (6\%). La Sous-préfecture de Soubré a enregistré le nombre de déclaration de décès le plus élevée

\section{III-3-1-3- Les PCC dans la déclaration des naissances et décès dans les délais}

Les Points de Collecte Communautaires ont été des acteurs techniques déterminants dans la collecte des données relatives aux naissances et décès dans tout l'environnement de l'étude. Ils méritent d'être redynamisés, promus et vulgarisés pour le succès des projets d'état civil à venir. En voici, une illustration statistique de l'impact pratique des PCC. 
Figure 2. Diagramme de synthèse des données des naissances et décès selon les $P C C$ et PCS

Naissances

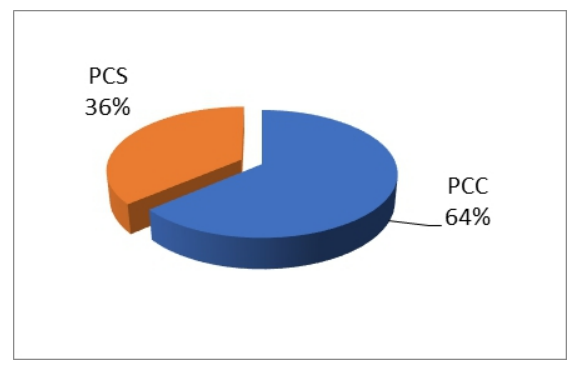

Décès

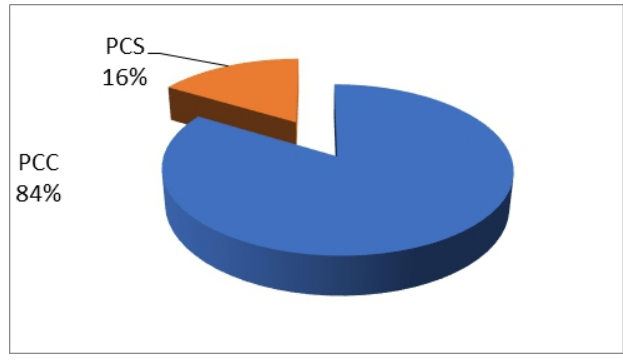

Source : Notre enquête de Février àmai, 2018

On constate à partir de ces diagrammes que les Points de Collecte Communautaires ont captés plus de données que les Points de Collecte Sanitaires. En dehors de la circonscription administrative de Soubré, ce constat est le même dans l'ensemble des huit (8) autres sous-préfectures. Pour les naissances, on note une différence de 2.220 soit 5.192 captées par les PCC contre 2.972 captées par les PCS. Cela représente 64\% de naissances pour les PCC contre $36 \%$ pour les PCS. Relativement aux décès, les PCC ont capté 133 décès soit $84 \%$ et les PCS 26 soit $16 \%$. Par ailleurs, on retient sur l'ensemble des données de naissances et décès collectées que, les nouveaux mécanismes permettent de capter beaucoup plus de naissances en communauté (dans les villages). En effet, les PCC, prennent en charge, dans le cadre de la mobilisation sociale, les parents (femme enceinte et son mari) depuis la prénatalité de la mère jusqu'à la déclaration à l'accouchement. Ce long contact régulier avec les parents augmente fortement les possibilités de voir tout nouveau-né être déclaré systématiquement par le PCC au cas où l'accouchement ne se produit par dans un centre sanitaire.

Toutefois, ce résultat acquis par les PCC met à nu un constat qui est celui du nombre encore élevé d'accouchements qui ont lieu dans les villages, en dehors des structures sanitaires. Cette situation s'explique par l'inaccessibilité (en termes de coût, distance et de temps) des populations rurales et des campagnes aux institutions sanitaires existantes. 


\section{III-3-2- De la considération de risques technologiques pour la pérénisation des nouveaux mécanismes d'enregistrement des naissances et décès dans les délais}

Figure 3. Courbes d'évolution des données des naissances et décès selon le mois

Courbe d'évolution des naissances

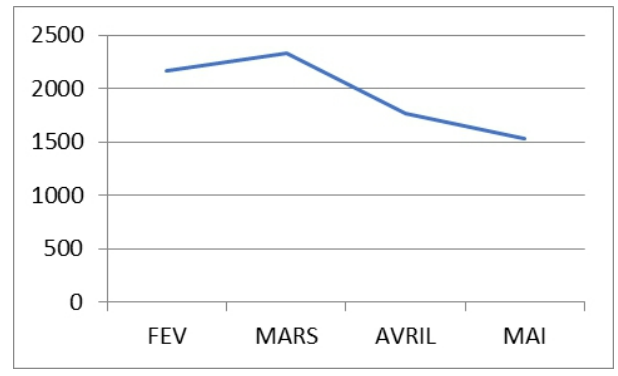

Courbe d'évolution des décès

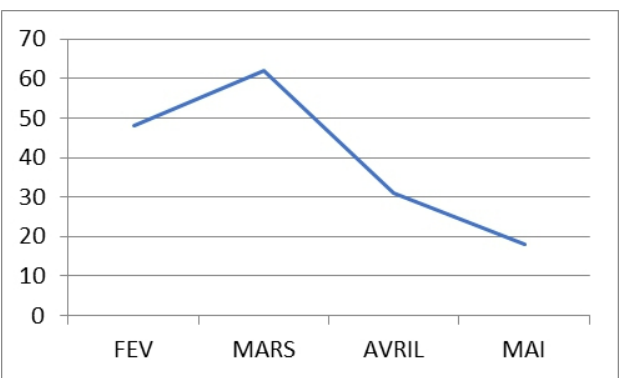

Source : Notre enquête de Février à Mai, 2018

Les deux courbes présentent l'évolution des données de naissances et de décès selon le mois précisément de février à mai. En observant ces courbes, l'on constate d'abord une croissante progressive au niveau des naissances et une croissance accélérée au niveau des décès les deux premiers mois (févriermars). Ensuite, on remarque une grande décroissance accélérée au niveau des décès jusqu'en avril suivie d'une autre progressivement jusqu'en mai. Au niveau des naissances, la courbe connait également une double décroissance mais équilibrée de mars en avril et d'avril en mai.

L'avènement des nouveaux mécanismes de déclaration à l'état civil a suscité beaucoup d'engouement et d'intérêt pour les populations qui ont été informée que l'administration de l'état civil venait désormais jusqu'à elle.

Cette situation, doublée de la motivation de départ des PCC et BEC, seraient à l'origine du nombre élevé de déclarations captées les premiers mois de l'opération (fév. et mars). Toutefois, l'absence de moyen de mobilité promis aux acteurs (vélos qui sont venus en retard), ainsi que l'absence de prime (ils travail en situation de bénévolat), ont occasionné des démotivations et des cas d'abandon de poste de certains agents sur le terrain. La décroissance qui s'est observée à partir du deuxième mois (Mars) de l'opération serait due à cette situation. Ainsi, de 2.330 déclarations en mars, l'on comptabilise 1.530 pour les naissances au mois de mai. Quant aux décès, de 62 déclarations en mars, l'on en compte 18 en mai. Cette décroissance des données collectées, conséquence des insuffisances de moyens d'accompagnement des acteurs des mécanismes innovants de déclaration de naissance et décès dans les délais, constitue l'ensemble des risques technologiques évoqués. Ainsi, les nouveaux mécanismes de déclaration des naissances et décès, malgré leur portée sociale, ont été impactés négativement par des facteurs d'ordre technonologique.Ceux-ci sont liés aux realités du phénomène de l'innovation 
en tant que processus de diffusion, d'adoption et d'adaptation avant son encrage social. En effet, lorque les moyens d'accompagnement d'une innovation technologique ne se deploientt pas progressivement, suivis continuellement et suffisants pour couvrir tout le procéssus d'innovation, cela constitue un facteur de risque pour le procéssus.

Par ailleurs, l'approche de sensibilisation utilisée par les PCC pour montrer aux femmes enceintes des zones rurales et des campagnes l'intérêt de l'accouchement dans les centres de santé n'a-t-elle pas constitué un des facteurs de cette décroissance dans la déclaration des naissances et décès ?

\section{III-3-3- L'importance de l'avènement des PCC et PCS dans la déclaration des naisances et décès dans les délais}

Soulignons d'emblée que les nouveaux mécanismes sont plus décisifs en matière de collectes de données de naissances et décès. en effet, l'étude comparative des données des naissances et décès des annuaires statistiques de 2014, 2015, 2016, 2017 avec les données collectées par les points de collecte communautaires (PCC) et points de collecte sanitaires (PCS) en 2018 a permis de confirmer cette hypothèse comme le montrent les statistiques sur cet état comparatif.

Tableau 2. Synthèse comparatif des données statistiques de la région de la

Nawa par sous-préfecture

Source : Notre enquête de Février à Mai, 2018

\begin{tabular}{|c|c|c|c|c|c|c|c|c|c|c|}
\hline \multirow[b]{4}{*}{ LOCALITES } & & & & & & & & & & \\
\hline & \multicolumn{10}{|c|}{ ETAT COMPARATIF } \\
\hline & \multicolumn{2}{|c|}{$\begin{array}{c}\text { DONNEES } \\
\text { DES PCC } \\
\text { ET PCS } \\
\text { (Février à Mai } \\
\text { 2018) }\end{array}$} & \multicolumn{2}{|c|}{$\begin{array}{l}\text { ANNUAIRE } \\
\text { STAT. } 2014 \\
\text { (Données } \\
\text { annuelles) }\end{array}$} & \multicolumn{2}{|c|}{$\begin{array}{l}\text { ANNUAIRE } \\
\text { STAT. } 2015 \\
\text { (Données } \\
\text { annuelles) }\end{array}$} & \multicolumn{2}{|c|}{$\begin{array}{l}\text { ANNUAIRE } \\
\text { STAT. } 2016 \\
\text { (Données } \\
\text { annuelles) }\end{array}$} & \multicolumn{2}{|c|}{$\begin{array}{l}\text { ANNUAIRE } \\
\text { STAT. } 2017 \\
\text { (Données } \\
\text { annuelles) }\end{array}$} \\
\hline & $\mathrm{N}$ & $\mathrm{D}$ & $\mathrm{N}$ & $\mathrm{D}$ & $\mathrm{N}$ & $\mathrm{D}$ & $\mathrm{N}$ & $\mathrm{D}$ & $\mathrm{N}$ & $\mathrm{D}$ \\
\hline SOUBRE & 790 & 29 & 3523 & 311 & 4487 & 341 & 3978 & 330 & 6816 & 245 \\
\hline GRAND ZATTRY & 742 & 10 & 2191 & 80 & 2580 & 168 & 2752 & 202 & 2745 & 179 \\
\hline LILIYO + MAYO & 889 & 19 & 756 & 15 & 876 & 19 & 838 & 24 & 1008 & 16 \\
\hline OKROUYO & 825 & 4 & 300 & 4 & 1108 & 0 & 1099 & 14 & 1040 & 19 \\
\hline MEAGUI & 1157 & 28 & 2563 & 44 & 2478 & 60 & 2231 & 79 & 2395 & 84 \\
\hline OUPOYO & 1248 & 27 & 1361 & 32 & 2612 & 22 & 1330 & 15 & 912 & 21 \\
\hline GUEYO & 571 & 3 & 1353 & 6 & 474 & 12 & 809 & 7 & 851 & 12 \\
\hline DABOUYO & 890 & 18 & 335 & 21 & 349 & 14 & 488 & 5 & 257 & 5 \\
\hline BUYO & 1052 & 21 & 1612 & 21 & 1806 & 31 & 1614 & 22 & 2790 & 47 \\
\hline $\begin{array}{l}\text { TOTAL NAISSANCES ET } \\
\text { DECES (NB: Délais et hors } \\
\text { délais pour les annuaires stat.) }\end{array}$ & 8164 & 159 & 13994 & 534 & 16770 & 667 & 15139 & 698 & 18814 & 628 \\
\hline $\begin{array}{c}\text { Déclaration dans les délais de } \\
\text { Février à Mai }\end{array}$ & 8164 & 159 & & & 4210 & 118 & 4044 & 200 & 5421 & 187 \\
\hline $\begin{array}{c}\text { Déclaration dans les délais } \\
\text { sur toute l'année }\end{array}$ & & & 13143 & & 14986 & 359 & 12757 & 661 & 15702 & 591 \\
\hline
\end{tabular}


Le constat général qui ressort est que, pour la même période de l'année (Fév. à Mai), l'instauration des nouveaux mécanismes a permis aux PCC et PCS de capter plus de 8.000naissances dans les délais représentant près du double des naissances dans les délais captés en 2015 et 2016 par le mécanisme habituel (ou ancien mécanisme). On note également une différence de 2.743 naissances captées de plus par les PCC et PCS entre 2018 et 2017. Les données captées par les nouveaux mécanismes représentent $37 \%$ de l'ensemble des données captées dans les délais de 2018, 2017, 2016 et 2015 pour la période de février à mai 2018.

Pour les décès, au regard des données captées pour la même période de février à mai, les résultats entre les nouveaux mécanismes en 2018 et les mécanismes habituels en 2017, 2016 et 2015 sont variables. Les nouveaux mécanismes représentent $24 \%$ en 2018 contre $28 \%$ en $2017,30 \%$ en 2016 et $18 \%$ en 2015.

On pourrait conclure que les nouveaux mécanismes n'ont pas encore permis de capter suffisamment les décès. Sur l'état comparatif, on peut noter que les nouveaux mécanismes à travers les PCC et PCS permettent de capter de manière significative les naissances. Enfin, les nouveaux mécanismes ont permis de relever de manière significative le nombre de déclaration de naissances dans les délais. Par contre, les décès sont encore insuffisamment captés.

\section{III-Discussion}

L'étude a revélé que l'introduction d'innovation dans les mécanimses de déclaration des naissances et décès dans les délais s'est avérée salutaire pour la plupart des polulations de la région de la Nawa (Soubré). Il s'agit en fait, d'une nouvelle expérience dans le système d'enregistrement des faits d'état civil en Côte d'Ivoire, qui à travers la captation éfficace par les PCC et PCS des naissances et décès dans les délais participe à sa transformation progressive. C'est dans ce contexte qu'en analysant le concept d'innovation, Saucier (2006) exprime l'idée selon laquelle l'innovation apparait donc comme un procéssus multiforme et multidimensionnel de production et de rénovation de l'existant, ou il importe d'nalyser les expérimentations qui prennent place dans les organisations innovantes, mais ou il importe tout autant de comprendre le processus institutionnel de reconnaissance et d'adoption, voir de diffusion et d'adaptation de l'innovation, qui provoquent la transformation sociale ou qui la donne à voir.Cette même réalité a été observée par Bayala et Ouedraogo (2011) qui proposent une nouvelle approche de collecte des données basée sur le système des fiches de collecte. Cette proposition a pour objectif de contribuer à la production de statistiques des faits de l'état civil au Burkina Faso. Ainsi, pour améliorer la collecte des statistiques de l'état civil afin de permettre une capitalisation des statistiques 
de l'état civil, les auteurs définissent l'instauration d'un registre d'état civil à quatre (4) volets pour chaque fait d'état civil avec les destinations suivantes: le premier volet reviendrait à l'individu (au déclarant), le second envoyé à l'institut de la statistique pour exploitation statistique, le troisième destiné à la justice pour les besoins de contrôle, le quatrième volet enfin reste dans le registre pour l'archivage. L'avantage de l'instauration de ces fiches se perçoit par le fait que l'individu accorde une considération vis à vis de l'enregistrement des faits de l'état civil. En outre, ces fiches combinent l'enregistrement dans les délais et celui hors délais. Abordant dans le même sens d'innovation des faits d'états civil par l'introduction de nouveaux mécanismes de collecte orientés vers les populations, Gogoua (2013) à travers l'expérience de son Organisation internationale pour l'enfant, la femme et la famille (ONG ONEF) dans le Département de Bondoukou, va aider à l'amélioration de l'enregistrement de nouvelles naissances grâce à une initiative novatrice portant sur la mise en place de nouveaux mécanismes dans ce département.

Il s'agit d'une part, du mécanisme communautaire de facilitation des déclarations des naissances (mis en place dans 69 quartiers et villages et animés par les populations elles-mêmes avec pour but de faciliter les déclarations de naissances dans les villages et quartiers ) fonctionnant sous la base de réunions mensuelles du mécanisme, et de points mensuels des activités au relais local; et d'autre part de la plateforme de suivi des déclarations de naissances (un mécanisme d'échanges, de partages, d'appui mutuel, d'assistance des populations et de plaidoyers entre les différents acteurs concernés par la déclaration des naissances au plan local) qui s'opérationnalise par la tenue des réunions trimestrielles, la rédaction de rapports annuels et trimestriels sur la déclaration des naissances et la recherche de solutions aux problèmes rencontrés sur le terrain. Contrairement à ces expériences innovatrices, réussies dans la déclaration des faits d'état civil, Paulet (1971) a montré que certaines normes culturelles et structurelles peuvent faire échouer l'innovation. A l'aide de l'exemple des deux cheikhats ruraux de Goraa et Oued-el-Khatef, l'auteur a souligné que l'un des premiers facteurs de la nondéclaration des naissances est le décès précoce, soit que celui-ci ait lieu avant que le père n'ait songé à déclarer la naissance, soit que ce dernier ait sciemment attendu d'être assuré de la survie de son enfant. Au niveau de la non-déclaration des décès, il s'agit du manque de temps des ménages, la négligence des administrés ou responsables locaux et la sévérité de la loi.

Par ailleurs, l'innovation dans les systèmes d'état civil peut se caractériser également par la combinaison de technologies nouvelles dans l'enregistrement et des statistiques comme par exemple, l'élaboration des lignes directrices sur l'intégration des systèmes de carte d'identité nationale et d'enregistrement des faits d'état civil pour faciliter la protection sociale et un 
développement plus inclusif (CRDI, 2018). Un cas similaire est révélé par les données de la quatrième Conférence des ministres africains chargés de l'enregistrement des faits d'état civil (APAI-CRVS, 2017) où le Mozambique est en train de mettre en place un système d'état civil modernisé qui documente tous les événements vitaux d'une personne et attribue un numéro unique à utiliser tout au long de la vie. Ceci ouvrira la voie à un système d'état civil ultramoderne de gestion de l'identification, relié au secteur de la santé et à l'Institut national de la statistique dans le cadre de l'initiative de gouvernance en ligne.

Dans ces conditions, on tend vers une innovation de plus en plus pointue dans l'enregistrement des faits d'état civil à telle enseigne que, cette nouvelle initiative de la Côte d'Ivoire, à travers le cas de la région de la Nawa (Soubré) par l'avènement des PCC, PCS, BEC doit être aussitôt promue et valorisée afin d'aboutir à de nouveaux mécanismes plus innovants et capables de rivaliser dans l'avenir, à l'échelle Africaine et Mondiale.

\section{Conclusion}

Cette étude a porté sur l'innovation technologique et sociale dans les mécanismes d'enregistrement des naissances et des décès dans les délais, dans la région de la Nawa (Soubré). Elle visait dans sa phase pratique, la recherche de mécanismes nouveaux pour tenter de résorber le problème du faible taux observé dans la déclaration des faits d'état civil. A l'issu d'une approche mixte (quantitative, qualitative) conduite à l'aide des techniques d'entretien, et de questionnaire, il importe de signifier le fort engagement créé chez la population qui voit désormais l'administration venir à elle au niveau local. Dans cette nouvelle dynamique ou l'institutionnel converge désormais vers le social, il faut noter les retombées positives que cette recherche a contribué à produire pour les populations en permettant la maitrise des naissances et décès survenant en temps réel dans les espaces sociaux de réalisation de l'étude. En effet, tous les acteurs y compris les populations bénéficiaires s'accordent à reconnaitre que le projet a été d'un grand bénéfice dans la mesure où le mouvement de l'administration vers les populations a favorisé la captation systématique des naissances et décès. Si quelques réticences subsistent encore quant à la déclaration des décès, il est à noter que la plupart des naissances sont captées tant par les PCC que les PCS.

Signalons toutefois que les PCC ont capté plus de données naissances et décès que les PCS. Ceci met à nu le nombre encore élevé d'accouchements en communautés (dans les villages) que dans les centres de santé. En rapprochant les centres de collecte des populations, le projet a permis aux populations d'éviter les nombreux déplacements et toutes autres formes de tracasseries dans la déclaration du fait d'état civil. 
$\mathrm{Au}$ regard du bilan positif observé en raison du nombre des déclarations qui a considérablement augmenté, la pérennisation du projet et son extension à l'échelle nationale est nécessaire afin de servir aux populations des autres zones. Dès lors, la prise en compte des préoccupations dans le cadre de cette étude s'avère primordiale pour assurer un réel succès du même projet dans sa phase de généralisation au grand bénéfice de nos populations. Toutefois, au regard des normes culturelles qui diffèrent d'une localité à une autre, la réalisation d'une étude sociologique sur les pesanteurs socioculturelles susceptibles d'échec n'est-elle pas indispensable avant la phase de généralisation de ce nouveau mécanisme de déclaration des faits d'état civil ?

\section{References:}

1. Bayala, L. \& Ouédraogo, G, F. (2011). Collecte des données de l'état civil : une nouvelle expérience au Burkina Faso. Sixième conférence internationale sur la population : population Africaine, passé, présent, futur. Session 15, question méthodologique, Séance 1551, approches innovatrices de collecte de données.

2. Centre de Recherches pour le Développement International (2018). Exploiter la puissance des systèmes d'enregistrement et de statistiques de l'état civil pour réaliser l'agenda 2030. Rapport sur les résultats de la conférence. Innovation, Ottawa, Canada.

3. APAI-CRVS (2017). Enregistrement des faits d'état civil : de la planification à la mise en œuvre. Conférence des Ministres Africain chargés de l'enregistrement des faits d'état civil, Nouakchott AUC/CRMC4/.

4. Dufour, C. \& Larivière, V. (2012). Principales techniques d'échantillonnage probabilistes et non-probabilistes. Sci6060fiche_echant.

5. Gogoua, R. (2013). «Construction des outils de repérage de défaut de déclaration des naissances des enfants, en particulier des fillettes. Cas de la Côte d'Ivoire ». Faciliter la déclaration des naissances : Echanges de bonnes pratiques, Parallel Event Regards de Femmes. $57^{\mathrm{eme}}$ Session CSW, ONG ONEF.

6. Marty, A.G. (1955). Analyse critique de l'œuvre de Joseph Shumpeter. Thèse pour doctorat. Université de Bordeau. Bruxelles : Ed. Montana. 250p.

7. Paulet, C. (1971). Les motifs du sous enregistrement des naissances et décès à l'état civil: une expérience tunisienne. Population, Le Maghreb. 26-1 pp. 245-249.

8. Projet d'appui au système national de planification et des statistiques (2015). Etude diagnostique de l'état civil en côte d'ivoire. Ministère 
d'état charge du plan et du développement, institut national de la statistique. Europe aïd/133467/ser/ci - agrer/afristat/ceso.

9. Rapport de l'opération projet pilote de promotion des nouveaux mécanismes de déclaration des naissances et décès dans les délais, région de la Nawa (2018). Ministère de l'intérieur et de la sécurité, office national d'identification.

10. Rapport stratégie nationale de l'état civil et de l'identification de la Côte d'Ivoire (2017). Ministère d'état, ministère de l'intérieur et de la sécurité.

11. Saucier Carol et al. (2006). «Développement et territoire», l'innovation sociale. Emergence et effets sur les transformations des sociétés, Juan-Luis Klein et Denis Harrisson (dir.), Québec, Presses de l'université du Québec, chapitre 19 pp. 337-395. 